\title{
Erratum to: Multiplicity of Solutions for Kirchhoff Fractional Differential Equations Involving the Liouville-Weyl Fractional Derivatives
}

\author{
Ali Ashraf Nori ${ }^{1}$, Nemat Nyamoradi ${ }^{* 2}$, and Nasrin Eghbali ${ }^{1}$ \\ ${ }^{1}$ University of Mohaghegh Ardabili, Ardabil, Iran \\ ${ }^{2}$ Razi University, Kermanshah, Iran
}

MSC2010 numbers : 34A08, 35A15

DOI: $10.3103 / \mathrm{S} 106836232004007 \mathrm{X}$

Third author, Nasrin Eghbali, is concerning to first institute.

The original article can be found online at

https://doi.org/10.3103/S1068362320010069

*Corresponding author E-mail: neamat80@yahoo.com 